\title{
Level I Evidence in Support of Perioperative Chemotherapy for Operable Gastric Cancer: Sufficient for Wide Clinical Use?
}

\author{
Evangelos Briasoulis, ${ }^{1}$ Michael Fatouros,${ }^{2}$ and Dimitrios H. Roukos ${ }^{2,3}$
}

\author{
${ }^{1}$ Medical Oncology, Ioannina University School of Medicine, Ioannina 45110, Greece \\ ${ }^{2}$ Department of Surgery, Ioannina University School of Medicine, Ioannina 45110, Greece \\ ${ }^{3}$ Surgical Oncology Research Unit, Department of Surgery, Ioannina University School of Medicine, Ioannina 45110, Greece
}

Primary surgery with gastrectomy and, if feasible, with extended D2 node dissection is the current standard treatment of operable gastric cancer., ${ }^{1,2}$ However, it is widely accepted that without adjuvant systemic treatment, the high relapse and death rates can hardly be improved. Despite research efforts, neither a chemotherapeutic regimen nor the timing of chemotherapy administration (i.e., before or after surgery) has been standardized. For the first time now, the MAGIC trial provides level I evidence for a survival benefit of perioperative chemotherapy over surgery alone in patients with localized gastric cancer. ${ }^{3}$ However, this study raises several questions: given that it was designed to assess an overall benefit and not a stage-specific survival benefit, it remains unknown as to which subgroups (II, IIIA or IIIB) mostly benefit from a perioperative regimen of epirubicin, cisplatin, and infused fluorouracil (ECF). Another key question is the impact of the MAGIC study in daily clinical practice. As several treatment options become available, uncertainty of oncologists for selecting an optimal adjuvant chemotherapy between perioperative, neoadjuvant and postoperative setting increases.

Gastric cancer is an aggressive malignancy with nearly 700,000 deaths annually (the second leading cause of cancer death) and a prevalence of around 930,000 new patients annually worldwide. ${ }^{4}$ Even the

Received November 30, 2006; accepted January 12, 2007; published online: July 26, 2007.

Address correspondence and reprint requests to: Dimitrios $\mathrm{H}$. Roukos; E-mail: droukos@cc.uoi.gr,info@gastricbreastcancer.com Published by Springer Science+Business Media, LLC @ 2007 The Society of Surgical Oncology, Inc. most appropriate local therapy, as an extensive D2/ D3 surgery, ${ }^{5}$ which increases ${ }^{6,7}$ the rate of a true complete surgical pathologic R0 resection, ${ }^{8}$ is associated with a substantial proportion of recurrence and death. ${ }^{9}$ Particularly in patients with advanced serosa-positive, node-positive gastric cancer, the 10year survival rate ranges between $10 \%{ }^{10}$ and, at best, $30 \% .^{5}$ Overall, less than $30 \%$ of Western patients are cured with local therapy alone as an R0 resection. ${ }^{10-13}$ Systemic adjuvant treatment is required for survival improvements.

The biological and clinical heterogeneity of gastric cancer represents a major challenge of current and future molecular and clinical research. ${ }^{14,15}$ Clinical data provide differential outcomes among patients with same TNM stage (I, II or III) and treatment ${ }^{7-9}$ and thus there is a clear requirement for tailoring adjuvant systemic therapy in treating specific subgroups of patients. ${ }^{15,16}$ Clinical models converted into a software program using conventional clinicopathologic prognostic factors have been developed and validated, ${ }^{17,18}$ but both these nomograms and the surgical pathologic TNM staging system have two major limitations: First, they cannot identify patients with local disease who could spare the toxic effects of unnecessary chemotherapy. Second, these conventional models cannot predict the response to certain chemotherapeutic regimens among patients with systemic disease who truly require adjuvant systemic treatment to reduce disease relapse and death rates.

In contrast to the proven efficacy of adjuvant cytotoxic chemotherapy in other adenocarcinomas including colorectal, lung and breast cancer, no conclusive data exist for gastric cancer. Many phase III clinical trials have explored this approach in 
gastric cancer but the survival benefit gained from the use of adjuvant chemotherapy in gastric cancer has not proven clinically significant ${ }^{16,19-23}$ and therefore adjuvant chemotherapy has not evolved as a part of the standard of care in patients with gastric cancer. $^{13,24}$

Level 1 evidence is considered the gold standard to be used for building treatment guidelines and recommendations. Therefore, the large-scale, phase III randomized MAGIC trial merits careful evaluation particularly considering its impact in clinical practice.

\section{MAGIC TRIAL: STRENGTHS, WEAKNESSES AND LIMITATIONS}

Cunningham et al. ${ }^{3}$ provide very important clinical information based on the data of a large-scale trial for a new therapy option in the treatment of gastric cancer. Perioperative chemotherapy should be distinguished from both neoadjuvant (preoperative) treatment, in which all adjuvant treatment protocol is applied before surgery, and postoperative, where treatment is administrated after surgery. In contrast to neoadjuvant treatment, pathological responses, either complete (pCR) or partial (pPR), to perioperative chemotherapy cannot be clearly assessed because part of the chemotherapy is administrated postoperatively. Thus, the only and major criterion of clinical effectiveness for perioperative strategy is clearance of disease and overall survival. In this phase III trial, 503 patients with resectable adenocarcinomas of the stomach, esophagogastric junction, or lower esophagus were randomly assigned to either perioperative chemotherapy (three preoperative and three postoperative cycles of ECF regimen) and surgery, or surgery alone (253 patients). The primary endpoint of the trial was overall survival.

The trial provided scientific evidence for improved outcomes with the use of the ECF regimen. The 5year overall survival rate was increased by $13 \%$ when perioperative chemotherapy was added to surgery. This improvement in survival corresponds to a $25 \%$ relative reduction in the risk of death (hazard ratio 0.75 ; 95\% confidence interval, 0.60 to 0.91 ; $p=0.009$ ). Progression-free survival also was improved by chemotherapy. Moreover, perioperative chemotherapy also achieved a decrease in tumor size and reduction in the extent of identified nodal metastases.

Despite the strengths, several intrinsic limitations and weaknesses of the MAGIC trial should be considered:

\section{(1) Generalizability, Lack of Tailoring Approach}

For many clinical trials, generalizability poses clinically important limitations. The MAGIC trial was designed to detect a significant difference in overall survival between ECF chemotherapy plus surgery over surgery alone for all tumor stages, II, IIIA and IIIB, together. Subgroup analysis was out of the scope of this study. Therefore, assessment of benefit according to tumor stage is difficult. Particularly for early stage serosa-negative cancer, the question of optimal treatment remains open. A recent Japanese multicenter randomized trial for serosa-negative cancer including node-positive disease showed the 5-year disease-free survival rate in the group receiving D2 surgery alone was $83 \%$ and the overall survival was $86 \%{ }^{25}$ Also, D2 surgery alone in Western patients with both serosa-negative and node-negative disease was associated with recurrence rates less than $10 \%^{10}$ and high 10 -year survival rates. ${ }^{12,26}$ These data show that many of patients diagnosed with, and treated for, early-stage cancer (local disease) are at low risk of recurrence, ${ }^{14}$ and can be cured with adequate surgical therapy alone. $^{27}$

Gene expression profiling of tumor tissue by means of microarrays and reverse-transcriptase polymerase chain reaction (RT-PCR) confirms the biologic and genetic heterogeneity of various cancer types including the most common breast, lung, gastric and other cancers. ${ }^{14,15,28-33}$ The heterogeneity of gastric cancer is also observed at a clinical level. Even in patients with the same TNM stage and similar clinical and pathologic features, the outcome varies: some are cured, whereas in others, the cancer recurs. ${ }^{1,15}$ Current conventional clinicopathologic staging is inadequate for predicting outcomes and response to chemotherapy. Recently, gene expression profiling studies have discovered and validated gene signatures, which are now commercially available for clinical use as biomarkers in breast cancer. ${ }^{30,33,34}$ Furthermore, large-scale trials for new targeted therapies to improve the response rates and survival for many cancer types have become new standards of treatment. ${ }^{34}$ These advances influence new gastric cancer research, but it should be made clear that none of these biomarkers or targeted drugs can be incorporated into clinical practice for gastric cancer management. At the present time only cytotoxic chemotherapy has been tested in large-scale randomized phase III clinical trials based on the current conventional staging methods. 


\section{(2) Completion of Treatment Protocol}

As Cunningham et al. ${ }^{3}$ note, only $42 \%$ of patients in the perioperative chemotherapy group completed protocol treatment; $34 \%$ of patients who completed preoperative chemotherapy and surgery did not begin postoperative chemotherapy, predominantly owing to early disease progression, patient request, or postoperative complications. Early disease progression under ECF regimen reflects the aggressive biology of gastric cancer and poses questions for the possibility to respond to other regimen.

Toxicity assessment is important when chemotherapy is tested. Cunningham et $\mathrm{al}^{3}{ }^{3}$ found that perioperative chemotherapy was associated with acceptable rates of adverse events. Excluding patients with neutropenia (23\%), less than $12 \%$ of patients suffered serious (grade 3 or 4 ) toxicity. Postoperative morbidity (45\%) and mortality (5.6\%) shown in the MAGIC trial are considered to be within an acceptable range as a Western trial, though they may be higher compared to those of trials from specialized institutions. ${ }^{25,35-37}$

\section{(3) New Chemotherapeutic Agents}

Cunningham and colleagues ${ }^{3}$ note that the utilized ECF regimen was developed in the late $1980 \mathrm{~s}^{38,39}$ and that there are now newer and less complex chemotherapy regimens with demonstrated activity against advanced gastric cancer. ${ }^{40}$ New studies will test newer regimens to assess whether these are better than ECF regimen in the perioperative setting.

\section{CHEMORADIATION}

Postoperative chemoradiation in gastric cancer with local and systemic effects presents an interesting therapeutic option. A large phase III trial of postoperative therapy strongly suggested a benefit from the combination of irradiation and chemotherapy after gastrectomy. ${ }^{41}$ This intergroup study 0116 (INT 0116), enrolled more than 550 patients who were randomly assigned to surgery alone or surgery followed by chemoradiation (fluorouracil and leucovorin plus external-beam radiation delivered to the tumor bed and areas of draining lymph nodes). These patients were at a significant risk for relapse after gastric resection - 85\% had lymph node metastases and $65 \%$ had stage T3 or T4 tumors. Median survival in the surgery only and surgery plus chemoradiation groups was 27 and 36 months, respectively
( $p=0.005$ by the log-rank test); the corresponding figures for disease-free survival were 19 and 30 months $(p<0.001)$. On the basis of these data, postoperative chemoradiation has been accepted as a standard of care in the USA among patients with resected gastric adenocarcinomas. ${ }^{13}$

However, toxicity was high and the positive results of this trial may be attributable to the efficacy of radiotherapy to control residual disease left by the inadequately limited surgery. Indeed, less than $10 \%$ of patients had D2 surgery, 54\% had D0 surgery and $36 \% \mathrm{D} 1$ surgery. ${ }^{41} \mathrm{D} 2$ surgery alone is the preferred treatment approach in specialized centers as it achieves better or similar local-regional control as D0/D1 surgery and chemoradiation ${ }^{5,12,42}$ with a lower side-effects profile. ${ }^{35-37}$ Despite all these limitations, postoperative chemoradiation makes an attractive option for countries and areas where D1 surgery is the primary treatment. In these centers, the oncologist's decision is based on recurrence risk estimates after surgery for associated surgical pathological tumor stage (pTNM). Risk of recurrence for node-positive and serosa-positive disease after limited surgery is high and can be reduced by chemoradiation. ${ }^{1}$

\section{CHALLENGES IN PRACTICE DECISIONS}

As it provides level 1 evidence, it is expected that the MAGIC study will influence the practice decisions of surgical oncologists. The trial was well designed and well conducted, and clinicians can have confidence in the findings of the study: Perioperatve ECF regimen improved survival by acceptable toxicity rates. However, given that stage-specific subgroup analysis was out of the scope of this study, the key question for clinicians is whether a decision to proceed with primary chemotherapy instead of primary surgery could harm more than benefit some of their patients. Given that patients with more advanced stages are at high risk of recurrence, it is logical to expect that these patients are those who would benefit the most from a perioperative therapeutic strategy. By contrast, for patients with early, serosa-negative cancer, who are at relatively low risk of recurrence, the optimal treatment remains to be defined. Indeed, these patients could avoid a delay in definitive surgery. Subsequently, decision on postoperative adjuvant chemotherapy could be based on exact surgical pathological staging (pT) taking the following into consideration: tumor depth, location, number of lymph nodes dissected and involved $(\mathrm{pN}$ 
stage), Lauren's histological type (intestinal, diffuse), and the extent of surgery (D1 or D2) performed. These pathological features, as well as clinical characteristics such age, can guide adjuvant treatment for patients at higher risk of recurrence. ${ }^{1}$

\section{CONCLUSIONS}

The MAGIC study showed that the perioperative ECF regimen, as compared with surgery alone, improved clinical outcomes of patients with localized stage II, IIIA and IIIB gastric cancer. After this robust finding, new studies are required to assess which stage-specific subgroup mostly benefits from adjuvant chemotherapy in the perioperative, neoadjuvant or postoperative setting.

Given the biological heterogeneity of gastric cancer it is not surprising that the current clinicopathologic staging methods and decisions on adjuvant treatment are suboptimal. Current research focused on molecular profiling of tumor tissue promises to discover and validate novel gene signatures that will improve recurrence risk stratification and predict response to various chemotherapeutic regimens. In addition to novel clinical biomarkers, new targeted therapies will further improve the clinical outcomes of patients with gastric cancer in the future.

\section{REFERENCES}

1. Roukos DH, Kappas AM. Perspectives in the treatment of gastric cancer. Nat Clin Pract Oncol 2005; 2:98-107.

2. NCI guidelines: http://www.nci.nih.gov/cancertopics/pdq/ treatment/gastric/healthprofessional/ Accessed 27 November 2006.

3. Cunningham D, Allum WH, Stenning SP, et al. Perioperative chemotherapy versus surgery alone for resectable gastroesophageal cancer. $N$ Engl J Med 2006; 355:11-20.

4. Parkin DM, Bray F, Ferlay J, Pisani P. Global cancer statistics, 2002. CA Cancer J Clin 2005; 55:74-108.

5. Sasako M. Principles of surgical treatment of curable gastric cancer. J Clin Oncol 2003; 21:274S-275S.

6. Roukos DH, Kappas AM. Targeting the optimal extent of lymph node dissection for gastric cancer. J Surg Oncol 2002; 81:59-62.

7. Roukos DH. Extended (D2) lymph node dissection for gastric cancer: do patients benefit?. Ann Surg Oncol 2000; 7:253-255.

8. Green FL, Page DL, Fleming ID, et al. (2002) Esophagus. In: Green FL, Page DL, Fleming ID (edset al.AJCC Cancer Staging Manual 6th ed.Springer-Verlag, New York, pp 91-98.

9. Roukos DH, Kappas AM. Limitations in controlling risk of recurrence after curative surgery for advanced gastric cancer are now well-explained by molecular-based mechanisms. Ann Surg Oncol 2001; 8:620-621.

10. Roukos DH, Lorenz M, Karakostas K, et al. Pathological serosa and node-based classification accurately predicts gastriccancer recurrence risk and outcome, and determines potential and limitation of a Japanese-style extensive surgery for Western patients. Br J Cancer 2001; 84:1602-1609.

11. Hartgrink HH, van de Velde CJ, Putter H, et al. Extended lymph node dissection for gastric cancer: who may benefit? Final results of the randomized Dutch gastric cancer group trial. J Clin Oncol 2004; 22:2069-2077.

12. Siewert JR, Bottcher K, Stein HJ, Roder JD. Relevant prognostic factors in gastric cancer: ten-year results of the German Gastric Cancer Study. Ann Surg 1998; 228:449-461.

13. Macdonald JS.. Gastric cancer - new therapeutic options. $N$ Engl J Med 2006; 355:76-77.

14. Roukos DH, Murray S, Briasoulis E Molecular genetic tools shape a roadmap towards a more accurate prognostic prediction and personalized management of cancer. Cancer Biol Ther 2007; 9:6(3) [Epub ahead of print].

15. Roukos DH, Liakakos T, Karatzas G, Kappas AM. Can VEGF-D and VEGFR-3 be used as biomarkers for therapeutic decisions in patients with gastric cancer?. Nat Clin Pract Oncol 2006; 3:418-419.

16. Briasoulis E, Liakakos T, Dova L, et al. Selecting a specific pre- or postoperative adjuvant therapy for individual patients with operable gastric cancer. Expert Rev Anticancer Ther 2006; 6:931-9.

17. Kattan MW, Karpeh MS, Mazumdar M, Brennan MF. Postoperative nomogram for disease-specific survival after an R0 resection for gastric carcinoma. J Clin Oncol 2003; 21:36473650 .

18. Novotny AR, Schuhmacher C, Busch R, Kattan MW, Brennan MF, Siewert JR. Predicting individual survival after gastric cancer resection: validation of a U.S.-derived nomogram at a single high-volume center in Europe. Ann Surg 2006; 243:7481.

19. Hermans J, Bonenkamp JJ, Boon MC, et al. Adjuvant therapy after curative resection for gastric cancer: meta-analysis of randomized trials. J Clin Oncol 1993; 11:1441-1447.

20. Mari E, Floriani I, Tinazzi A, et al. Efficacy of adjuvant chemotherapy after curative resection for gastric cancer: a metaanalysis of published randomised trials. Ann Oncol 2000; 11:837-843.

21. Janunger KG, Hafstrom L, Glimelius B. Chemotherapy in gastric cancer: a review and updated meta-analysis. Eur J Surg 2002; 168:597-608.

22. Earle CC, Maroun JA. Adjuvant chemotherapy after curative resection for gastric cancer in non-Asian patients: revisiting a meta-analysis of randomised trials. Eur J Cancer 1999; 35:1059-1064.

23. Panzini I, Gianni L, Fattori PP, et al. Adjuvant chemotherapy in gastric cancer: a meta-analysis of randomized trials and a comparison with previous meta-analyses. Tumori 2002; 88:21-27.

24. Roukos DH. Current status and future perspectives in gastric cancer management. Cancer Treat Rev 2000; 26:243-255.

25. Nashimoto A, Nakajima T, Furukawa H, et al. Randomized trial of adjuvant chemotherapy with mitomycin, Fluorouracil, and Cytosine arabinoside followed by oral Fluorouracil in serosa-negative gastric cancer: Japan Clinical Oncology Group 9206-1. J Clin Oncol 2003; 21:2282-2287.

26. Roukos DH. Current advances and changes in treatment strategy may improve survival and quality of life in patients with potentially curable gastric cancer. Ann Surg Oncol 1999; 6:46-56.

27. Roukos DH. Early-Stage Gastric cancer - A highly treatable disease. Ann Surg Oncol 2004; 11:127-129.

28. Ramaswamy S., Ross KN., Lander ES. A molecular signature of metastasis in primary solid tumours. Nat Genet 2003; 33:49-54.

29. van't Veer LJ, Dai H, van de Vijver MJ, et al. Gene expression profiling predicts clinical outcome of breast cancer. Nature 2002; 415:530-536.

30. van de Vijver He M. J. YD, van't Veer LJ, et al. A geneexpression signature as a predictor of survival in breast cancer. N Engl J Med 2002; 347:1999-2009. 
31. Potti A, Mukherjee S, Petersen R, et al. A genomic strategy to refine prognosis in early-stage non-small-cell lung cancer. $N$ Engl J Med 2006; 355:570-580.

32. Perou CM, Sorlie T, Eisen MB, et al. Molecular portraits of human breast tumours. Nature 2000; 406:747-752.

33. Paik S, Shak S, Tang G, et al. A multigene assay to predict recurrence of tamoxifen-treated, node-negative breast cancer. $N$ Engl J Med 2004; 351:2817-2826.

34. Ozols RF, Herbst RS, Colson YL, et al. Clinical cancer advances 2006: major research advances in cancer treatment, prevention, and screening-a report from the American Society of Clinical Oncology. J Clin Oncol 2007; 25:146-162.

35. Degiuli M, Sasako M, Calgaro M, et al. Morbidity and mortality after D1 and D2 gastrectomy for cancer: interim analysis of the Italian Gastric Cancer Study Group (IGCSG) randomised surgical trial. Eur J Surg Oncol 2004; 30:303-308.

36. Wu CW, Hsiung SA, Lo, et al. Nodal dissection for patients with gastric cancer: a randomised trial. Lancet Oncol 2006; 7:309-315.

37. Sano T, Sasako M, Yamamoto S, et al. Gastric cancer surgery: Morbidity and mortality results from a prospective randomized controlled trial comparing D2 and extended para-aortic lymphadenectomy-Japan Clinical Oncology Group Study 9501. J Clin Oncol 2004; 22:2767-2773.

38. Findlay M, Cunningham D, Norman A, et al. A phase II study in advanced gastro-esophageal cancer using epirubicin and cisplatin in combination with continuous infusion 5-fluorouracil (ECF). Ann Oncol 1994; 5:609-616.

39. Webb A, Cunningham D, Scarffe JH, et al. Randomized trial comparing epirubicin, cisplatin, and fluorouracil versus fluorouracil, doxorubicin, and methotrexate in advanced esophagogastric cancer. J Clin Oncol 1997; 15:261-267.

40. Sumpter K, Harper-Wynne C, Cunningham D, et al. Report of two protocol planned interim analyses in a randomised multicentre phase III study comparing capecitabine with fluorouracil and oxaliplatin with cisplatin in patients with advanced esophagogastric cancer receiving ECF. Br J Cancer 2005; 92:1976-1983.

41. Macdonald JS, Smalley SR, Benedetti J, et al. Chemoradiotherapy after surgery compared with surgery alone for adenocarcinoma of the stomach or gastroesophageal junction. $N$ Engl J Med 2001; 345:725-730.

42. Roukos . Adjuvant chemoradiotherapy in gastric cancer: wave goodbye to extensive surgery?. Ann Surg Oncol. 2002; 9:220 221.

43. Roukos DH, Lorenz M, Encke A. Evidence of survival benefit of extended (D2) lymphadenectomy in western patients with gastric cancer based on a new concept: a prospective long-term follow-up study. Surgery 1998; 123:573-578.

44. Kappas AM, Fatouros M, Roukos DH. Is it time to change surgical strategy for gastric cancer in the United States?. Ann Surg Oncol 2004; 11:727-730. 